\title{
Successful aortic valve replacement in Leptospira infection
}

\author{
Szabó $Z^{1 *}$, Alotti ${ }^{2}$, Szélig $L^{1}$, Magyar $K^{3}$, Loibl $C^{1}$, Frenyó $M^{2}$, Csécs $R^{2}$, Bogár $L^{1}$ and Csontos $C^{1}$ \\ ${ }^{1}$ Department of Anaesthesiology and Intensive Care, University of Pécs, Hungary \\ ${ }^{2}$ Szent Raphael Country Hospital, Zalaegerszeg, Hungary \\ ${ }^{3}$ First Department of Internal Medicine, University of Pécs, Hungary
}

\begin{abstract}
We present a young male patient's case of severe aortic valve stenosis complicated with acute Leptospira infection. Stenosis had caused circulatory decompensation; therefore, patient was scheduled for elective cardiac surgery. One month before operation Leptospira infection was diagnosed. With literature search aortic valve replacement was performed at the earliest 3 months after such infection. In our case, we could not wait so long. Soon after the resolution of septicemic phase successful aortic valve replacement was performed. An uneventful postoperative period followed, and patient was discharged home. In this case report the diagnostic and therapeutic problems are described.
\end{abstract}

\section{Introduction}

Leptospirosis is one of the most common antropozoonosis worldwide. Its symptomatology is rather complex. Numerous specific and non-specific clinical appearances of leptospirosis are known, which could explain the failure of diagnosis [1]. The animals that spread the disease (rats, rodents, pigs, dogs) can urinate it throughout their whole life without falling ill. The pathogen can get into the human body through the conjunctiva, mucosa, and skin injuries [2]. The disease has two stages: acute septicemic (days 4 to 9 ) and immune phase (days 9 to 30). Leptospirosis with hepatorenal involvement is called Weil's syndrome. In this case the course of the disease is expected to be serious. Kidney involvement (acute tubular necrosis (ATN), nephritis), clouding of consciousness, serous meningitis, and high fever can appear in addition to icterus moreover hemorrhagic pneumonia, and serious circulatory insufficiency can develop beside liver failure [3]. In these severe cases, death rate can be $2-40 \%$ despite antibiotic therapy. Numerous observations have proven that chronic leptospirosis does not exist. Even the most serious infections can be cured without remnant symptoms [2].

\section{Case report}

In the anamnesis of the 43-year-old male patient serious aortic stenosis could be found. For this reason, in July 2016 valve surgery was indicated. The echocardiographic examination showed calcified aortic valve with limited movement. Furthermore, the examination showed that the peak gradient was $112 \mathrm{mmHg}$ and the average gradient was 77 $\mathrm{mmHg}$. The cardiac compensated patient, who was free of complaints, was then booked for focus examination and coronarography.

The examination was carried out in April 2017. At the end of the examination, no focuses were found furthermore; during the coronarography significant stenosis was not proven. The ECHO examination showed seriously degenerated aortic valve. The average gradient of the aortic stenosis was $80 \mathrm{mmHg}$. Grade I aortic insufficiency and diffusely reduced left ventricular wall motion were noted. At this point, the patient complained of low load level, asphyxia, coughing, and lack of appetite. Drug therapy was started (furosemide, potassium, ramipril, metoprolol, pantoprazole), and by the end of May, he was booked for valve surgery and the patient was discharged.

The next day he was taken to the Department of Emergency Medicine because of faint. He suffered from hypotension and tachypnea. Blood gas testing showed metabolic acidosis. In the lab tests liver and kidney failure were found. Between the liver and the diaphragm, a 1 $\mathrm{cm}$ thick, in the true pelvis an approximately $3 \mathrm{~cm}$ free abdominal fluid was detected by abdominal ultrasound examination. The circulation of vena portae was moderately slow. The general condition of the patient further deteriorated under conservative therapy. Considering that the patient suffered from acute liver failure and a liver replacement therapy may be necessary, the patient was transferred to our intensive care unit. Sampling for serology was carried out prior to patient transfer (hepatitis A (HAV), hepatitis B (HBV), hepatitis C (HVC), toxoplasma). The toxicology examination did not reveal a clear agent. Liver failure caused by cardiac decompensation was ruled out because of the fulminant course of the disease.

When the patient arrived, his vital parameters were stable (blood pressure $100 / 80 \mathrm{mmHg}$, heart rate $83 / \mathrm{min}$, peripheral hemoglobin oxygen saturation $100 \%$ with $30 \%$ oxygen). He was alert, conscious and diuresis were normal. Arterial and high flow central venous cannulae were inserted. Considering the fulminant liver failure of the patient, a mega dose of acetylcysteine was initiated. In the lab findings on admission, a further decrease in serum sodium and an increase in INR and renal function values were found. To stabilize his coagulation 1500 IU Beriplex ${ }^{\oplus}$ and 4 IU fresh frozen plasma were given. We requested for a gastroenterological consultation, which suggested Leptospira, HAV, $\mathrm{HBV}, \mathrm{HCV}$, hepatitis $\mathrm{E}$ (HEV) virus serology, paracetamol and fungus toxin examination, and abdominal imaging study. The patient negated the consumption of any toxin (medicine, fungus, alcohol, drug). The

*Correspondence to: Zsófia Szabó, Department of Anaesthesiology and Intensive Care, University of Pécs, Hungary, E-mail: zsofiaeszter.szabo@gmail.com

Key words: Leptospira infection, invasive monitoring, aortic valve replacement

Received: June 04, 2018; Accepted: June 18, 2018; Published: June 22, 2018 
CT examination revealed serious circulatory disorder in the liver, and suggested a supplementary abdominal ultrasound examination, which did not confirm it. Considering the earlier cardiac condition of the patient, ECHO examination was requested. In the echocardiography examination the following were shown: ventricular ejection fraction (EF): $30 \%$, wide left ventricular cavity size, calcified aortic valves, serious aortic stenosis (AS) (area: $0.8 \mathrm{~cm}^{2}$ ), severe aortic insufficiency (AI), pressure half time (PHT): $140 \mathrm{~ms}$ ), and elevated right ventricular pressure. The cardiologist recommended cardiac surgery consultation. Infective endocarditis was thought to be behind the picture revealed by the ECHO examination, and the elevated inflammation parameters. Thus, a series of blood cultures were taken. Because of the elevated right ventricular pressure, CT examination was requested (related to pulmonary embolism), which confirmed pulmonary embolism and infarct pneumonia. The patient was referred to a heart surgeon, who thought that surgery was not necessary. Because of the general deterioration in the condition of the patient and laboratory parameters, a continuous renal replacement therapy (CRRT) was initiated on the day of the patient's arrival. The gastroenterological consultation was repeated. According to the consultation, the reason for the elevated creatine-kinase and stasis was cardiologic not hepatologic, but the laboratory indicated to us that, based on the urine sample, the patient may be infected by Leptospira. For this reason, we consulted with an infectologist, and decided on amoxicillin-clavulanic acid treatment. After 24 hours the CRRT had to be stopped because the filter became clotted. The inflammatory parameters and the liver and renal function values of the patient showed decreasing tendency, so CRRT was not restarted. On the next day Leptospira infection was confirmed by PCR.

Four days after the admission of the patient, his general condition deteriorated. He was complaining about intensive hiccup and asphyxia. As his gas exchange was not satisfactory with $100 \%$ oxygen we decided on nasal high flow $\left(\mathrm{FiO}_{2}\right.$ : 0.6; $60 \mathrm{l}$ /minute flow) oxygen therapy. We asked for another cardiologic consultation, which revealed seriously stenotic aortic valve and the intensification of aortic regurgitation compared to the previous condition of the patient. Urgent bedside cardiac surgery consultation and acute valve surgery were suggested. At this point, because of the verified Leptospira infection and the urgent valve surgery, we asked for an infectologist consultation. The infectologist suggested that the patient should change antibiotics (we started with imipenem). Cardiac surgeons then again advised to postpone heart surgery. To judge the cardiac and hemodynamic status of the patient, we started invasive hemodynamic monitoring (PiCCO). As the results suggested overfilling, we decided to start CRRT and dobutamine treatment. The patient started to urinate, his liver enzymes normalized, bilirubin greatly decreased, and practically his coagulation status normalized, so CRRT was stopped on the sixth day. With $30 \% \mathrm{O}_{2}$ the blood gas parameters of the patient were in the normal range.

By the next day, his cardiac function index (CI): dropped to 1.5 $1 /$ minute $/ \mathrm{m}^{2}$ - levosimendan treatment was started. His hourly urine output improved. CI rose above $2.5 \mathrm{l} / \mathrm{min} / \mathrm{m}^{2}$. A new CRRT was not necessary. We asked for acute heart surgery consultation related to ECMO treatment. Considering the unfavorable liver function values and positive inflammatory parameters, the risk of ECMO treatment was considered high, thus it was not suggested.

On the third day of the levosimendan treatment, the inflammatory parameters of the patient were rising, he became sub febrile. There was again a chance of infectious endocarditis. Transesophageal echocardiography (TEE) showed serious valve destruction with suspicion of rupture, aortic stenosis, aortic regurgitation (III-IV) and apposition referring to infectious endocarditis could be seen (Figure 1). The heart surgery consultation suggested that the patient should be taken to the cardiovascular surgery department where he was scheduled for operation. The patient was admitted on the next day.

At admission, signs of circulation insufficiency were noted. His blood pressure was in the normal range with positive inotrope medication. After a short preparation, the patient underwent surgery immediately with cardiopulmonary bypass (CPB). During the surgery, broadened left ventricle, congestive right ventricle and bilateral hydrothorax were found. The surgical findings showed a very calcified aortic valve with a narrow orifice without any sign of inflammation (Figure 2). Under the valve, concentric limestone blocks penetrating into the muscles of the left ventricle could be seen. The intima of the ascending aorta was interspersed with calcific plaques. After the removal of the aortic valve and the sub valvular calcific deposits, mechanic artificial valve was implanted. In the first 15 hours of the postoperative period, combined catecholamine support was needed. With stable respiratory and circulatory parameters, the patient could be extubated after 12 hours. His antibacterial treatment contained ceftriaxone in addition the usual perioperative endocarditis prophilaxys according to the original pathogen. Because of grade III AV block, DDD PM implantation was carried out on the sixth postoperative day. The TTE examination on the sixth postoperative day showed broad hypertrophic left ventricle, diffuse hypokinesis, good artificial aortic valve function without paravalvular leak (EF: $45 \%$ peak gradient $16 \mathrm{mmHg}$ ).

On the eighth postoperative day, the patient was transferred to a cardiac rehabilitation department. From here, he was released after three weeks with compensated respiratory and circulatory parameters.

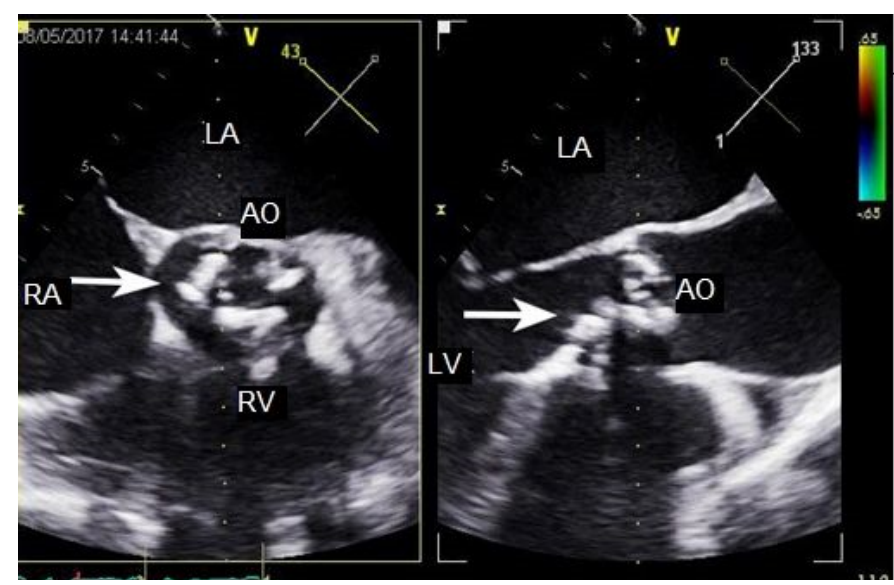

Figure 1. Transoesophageal echocardiography. Arrows indicate calcified aortic valve $\mathrm{RV}$ : right ventricle, $\mathrm{AO}$ : aortic valve, RA: right atrium, LA: left atrium

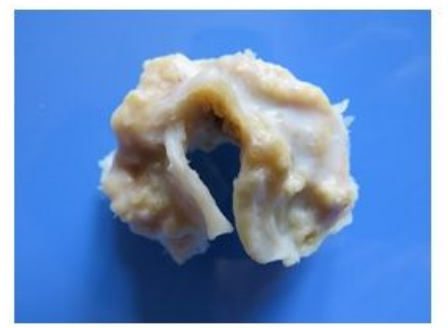

Left ventricle view

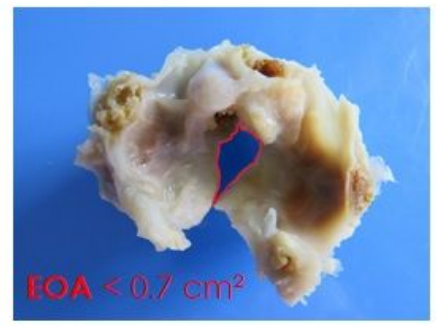

Aortic view
Figure 2. Excided aortic valve. EOA: estimated orifice area 


\section{Discussion}

The difficulty of the case was find out the origin of the liver and renal failure. We could find only one literature on aortic valve replacement where the patient also suffered from Leptospira infection. In this case, doctors waited for two months before the surgery [4].

Positive results during the treatment of the patient were likely to indicate Leptospira infection behind the liver and renal failure. However, neither the Leptospira infection nor the pulmonary embolism could not provide any explanation for the right ventricular insufficiency, but the improving status of the patient after the antibiotics therapy and CRRT suggested the role of Leptospira. Nevertheless, the repeated episodes of decompensation, the deterioration of the $\mathrm{ECHO}$ picture tended to show decompensated aortic stenosis. From this time on, the main difficulty was to choose the right time of the surgery. Based on the literature, we wanted to prolong the surgery by two months, however, the repeated uncontrollable decompensations did not allow us to do so. Besides the CRRT, dobutamine and levosimendan treatment, there were no other possibilities of conservative therapy. We may have tried ECMO therapy, but according to the heart surgeon, the risks of this treatment would have been the same as that of the surgery. With conservative treatment 15 days passed between the admission of the patient and the heart surgery. Thus, the successful acute surgery took place in the immune phase, and not in the septicotoxemic phase of the Leptospira infection.

We declare that the patient gave an informed consent.

\section{References}

1. Costa F, Hagan JE, Calcagno J, Kane M, Torgerson P, et al. (2015) Global Morbidity and Mortality of Leptospirosis: A Systematic Review. PLoS Negl Trop Dis 9: e0003898 [Crossref]

2. Haake DA, Levett PN (2015) Leptospirosis in humans. Curr Top Microbiol Immunol 387: 65-97. [Crossref]

3. Aslan Ö (2015) A review on the clinical outlook of leptospirosis. Inter Res J Emerg Trends Multidisc 50-55.

4. Butler CS, Endara SA (2000) Leptospirosis complicated by severe aortic stenosis. Anaesth Intensive Care 28: 434-437. [Crossref]

Copyright: (C2018 Szabó Z. This is an open-access article distributed under the terms of the Creative Commons Attribution License, which permits unrestricted use, distribution, and reproduction in any medium, provided the original author and source are credited. 